\title{
The Schwinger formula revisited II (A Mathematical Treatment)
}

\author{
Jaume Haro
}

October 2003

Departament de Matemàtica Aplicada I, Universitat Politècnica de Catalunya, Diagonal 647, 08028 Barcelona, Spain.

e-mail: jaime.haro@upc.es

\begin{abstract}
In this paper we study the production of pairs in no-analytic potentials. It is a well-known fact that, when the potential is analytic the average number of produced pairs is exponentially small in $\hbar$. On the other hand, when the potential is no-analytic, using the W.K.B. method, we prove that the average number of produced pairs is $O\left(\alpha \hbar^{2 N}\right)$, where $N$ is the regularity of the potential and $\alpha$ is the fine structure constant. Finally, we give a rigorous proof of the Schwinger's formula.
\end{abstract}

Key words. Pair production, Schwinger's formula, W.K.B. method.

AMS subject classifications. 34E20, 81Q05, 81Q20. 


\section{Contents}

1 Introduction $\quad 3$

2 Production of particles without spin $\quad 3$

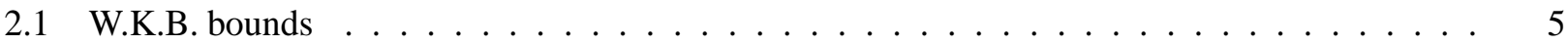

3 Application of the W.K.B. method $\quad 7$

3.1 Computation of the number of produced pairs . . . . . . . . . . . . . . . . 9

$\begin{array}{llr}\text { The Schwinger formula } & 10\end{array}$

5 References $\quad 16$ 


\section{Introduction}

In the previous paper (Haro, to appear) we have obtained the formula that give the average number of produced pairs due to the presence of an external uniform field. The most important application of this formula is the "formal" deduction of the average number of produced pairs in a constant electric field.

Here, the objective is the study of the average number of produced pairs in no-analytic fields (the case of an analytic potential has studied in (Eisenberg and Kälberman, 1988; Marinov and Popov, 1977; Popov, 1972)) and the rigorous computation of the average number of produced pairs in a constant electric field.

First, we will see the relation that exists between the probability that a pair is created and the transmission and reflection coefficients of the associated Klein-Gordon equation. It is well-known (Berry, 1982; Fulling, 1985) that these coefficients depend of the regularity of the field, thus, the average number of produced pairs depends of the regularity of the field, this fact is explained in detail in section 2 .

In section 3, we give bounds of the average number of produced pairs in an uniform electric field that have regularity $\mathcal{C}^{N-1}$. For this propose, we will use the W.K.B. method, in a similar way used in (Berry, 1982), and we give bound of the error that we obtain using this method. We prove that the average number of produced pairs is $O\left(\alpha \hbar^{2 N}\right)$, where $\alpha$ is the fine structure constant. And we see that, in the case that the electric field has a discontinuity at the point $T$, the average number of produced pairs, when the field is switched off, is in the semi-classical approximation

$$
\frac{\alpha E(T)}{64 m c^{2}}
$$

where $E(T)$ is the energy of the field at time $T$.

Finally in Section 4, we prove that the average number of produced pairs per unit time and unit volume in a constant electric field, when the time increases to infinity, is

$$
\frac{E^{2} \alpha}{8 \pi^{3} \hbar} \exp \left(-\frac{\pi m^{2} c^{4}}{\hbar c e E}\right)
$$

This result has formally proved for some authors (Haro, to appear; Holstein, 1999; Nikishov, 1970). In this work we give a demonstration that is very large and complicated, because we use the asymptotic expressions of the hipergeometric confluent functions with the bounds of the remainding terms, and also a semi-classical solution of the second quantized Klein-Gordon field equation, in the Schödinger picture, is used. But any way, we believe that a rigorous demonstration and a suitable interpretation of this result was needed.

\section{Production of particles without spin}

As in the previous paper, we consider the Klein-Gordon field in a box of volume $L^{3}$, coupled with an external uniform vector potential $\vec{f}(t)$. The Hamilton equations are

$$
\ddot{u}_{\vec{k}}+\omega_{\vec{k}}^{2}(t) u_{\vec{k}}=0, \quad \vec{k} \in \mathbb{Z}^{3},
$$

with

$$
\omega_{\vec{k}}^{2}(t)=\frac{1}{\hbar^{2}}\left(c^{2}\left|\frac{2 \pi \hbar \vec{k}}{L}+\frac{e}{c} \vec{f}(t)\right|^{2}+m^{2} c^{4}\right)
$$


We suppose that $\omega_{\vec{k}}(t)$ has the following form

$$
\omega_{\vec{k}}(t)=\left\{\begin{array}{ccc}
\omega_{1, \vec{k}} & \text { if } & t<T_{1} \\
\omega_{\vec{k}}(t) \quad \in \mathcal{C}^{\infty}\left(T_{1}, T_{2}\right) & \text { if } & T_{1}<t<T_{2} \\
\omega_{2, \vec{k}} & \text { if } & t>T_{2}
\end{array}\right.
$$

We look solutions of (1) in the following form

$$
u_{\vec{k}}(t)=\left\{\begin{array}{ccc}
\phi_{1, \vec{k}}^{+}(t) & \text { if } & t<T_{1} \\
A_{\vec{k}} \varphi_{\vec{k}}^{+}(t)+B_{\vec{k}} \varphi_{\vec{k}}^{-}(t) & \text { if } & T_{1}<t<T_{2} \\
a_{\vec{k}} \phi_{2, \vec{k}}^{+}(t)+b_{\vec{k}} \varphi_{2, \vec{k}}^{-}(t) & \text { if } & t>T_{2},
\end{array}\right.
$$

where

$$
\phi_{\left(\begin{array}{l}
1 \\
2
\end{array}\right)}^{ \pm}(t)=\frac{\exp \left( \pm i \omega_{\left(\begin{array}{l}
1 \\
2
\end{array}\right), \vec{k}}\left(t-T_{\left(\begin{array}{l}
1 \\
2
\end{array}\right)}\right)\right)}{\sqrt{2 \omega_{\left(\begin{array}{l}
1 \\
2
\end{array}\right), \vec{k}^{\hbar}}}} .
$$

Therefore, the average number of produced pairs in the $\vec{k}$-state at time $t>T_{2}$ is (Fulling, 1985)

$$
N_{\vec{k}}\left(t>T_{2}\right)=\left|b_{\vec{k}}\right|^{2}
$$

and the total average number of produced pairs at time $t>T_{2}$, is

$$
N\left(t>T_{2}\right)=\sum_{\vec{k} \in \mathbb{Z}^{3}}\left|b_{\vec{k}}\right|^{2}
$$

In order to calculate $a_{\vec{k}}$ and $b_{\vec{k}}$, we suppose that $u_{\vec{k}}(t) \in \mathcal{C}^{1}(\mathbb{R})$, then we obtain

$$
\begin{array}{cc}
a_{\vec{k}}=\frac{i \hbar}{W\left[\varphi_{\vec{k}}^{+}\left(T_{1}^{+}\right), \varphi_{\vec{k}}^{-}\left(T_{1}^{+}\right)\right]} \quad\left[W\left[\phi_{1, \vec{k}}^{+}\left(T_{1}^{-}\right), \varphi_{\vec{k}}^{-}\left(T_{1}^{+}\right)\right] W\left[\varphi_{\vec{k}}^{+}\left(T_{2}^{-}\right), \phi_{2, \vec{k}}^{-}\left(T_{2}^{+}\right)\right]\right. \\
& \left.-W\left[\phi_{1, \vec{k}}^{+}\left(T_{1}^{-}\right), \varphi_{\vec{k}}^{+}\left(T_{1}^{+}\right)\right] W\left[\varphi_{\vec{k}}^{-}\left(T_{2}^{-}\right), \phi_{2, \vec{k}}^{-}\left(T_{2}^{+}\right)\right]\right] \\
b_{\vec{k}}=\frac{-i \hbar}{W\left[\varphi_{\vec{k}}^{+}\left(T_{1}^{+}\right), \varphi_{\vec{k}}^{-}\left(T_{1}^{+}\right)\right]} \quad\left[W\left[\phi_{1, \vec{k}}^{+}\left(T_{1}^{-}\right), \varphi_{\vec{k}}^{-}\left(T_{1}^{+}\right)\right] W\left[\varphi_{\vec{k}}^{+}\left(T_{2}^{-}\right), \phi_{2, \vec{k}}^{+}\left(T_{2}^{+}\right)\right]\right. \\
\left.-W\left[\phi_{1, \vec{k}}^{+}\left(T_{1}^{-}\right), \varphi_{\vec{k}}^{+}\left(T_{1}^{+}\right)\right] W\left[\varphi_{\vec{k}}^{-}\left(T_{2}^{-}\right), \phi_{2, \vec{k}}^{+}\left(T_{2}^{+}\right)\right]\right],
\end{array}
$$

where $W[f(t), g(t)]$ is the Wronskian of the functions $f$ and $g$ at the point $t$, and

$$
f\left(T^{+}\right)=\lim _{\substack{t \rightarrow T \\ t>T}} f(t) ; \quad f\left(T^{-}\right)=\lim _{\substack{t \rightarrow T \\ t<T}} f(t)
$$


Remark 2.1. As a consequence of the constancy of the Wronskian, we have $\left|a_{\vec{k}}\right|^{2}-\left|b_{\vec{k}}\right|^{2}=1$.

Remark 2.2. If we take $\varphi_{\vec{k}}^{ \pm}$such that

$$
\left\{\begin{array}{l}
\phi_{1, \vec{k}}^{ \pm}\left(T_{1}^{-}\right)=\varphi_{\vec{k}}^{ \pm}\left(T_{1}^{+}\right) \\
\dot{\phi}_{1, \vec{k}}^{ \pm}\left(T_{1}^{-}\right)=\dot{\varphi}_{\vec{k}}^{ \pm}\left(T_{1}^{+}\right)
\end{array}\right.
$$

Then, we obtain the following expression

$$
\left|b_{\vec{k}}\right|^{2}=\hbar^{2}\left|W\left[\varphi_{\vec{k}}^{+}\left(T_{2}\right), \phi_{2, \vec{k}}^{+}\left(T_{2}\right)\right]\right|^{2}
$$

For this reason, we will always demand that the condition (10) is satisfied.

Now, in order to calculate $\varphi_{\vec{k}}^{ \pm}(t)$, we use the W.K.B. method. We write (Berry, 1982)

$$
\varphi_{\vec{k}}^{ \pm}(t)=\frac{1}{\sqrt{2 \epsilon_{\vec{k}}\left(T_{1}\right)}} \exp \left( \pm \frac{i}{\hbar} \int_{T_{1}}^{t} P_{\vec{k}}^{ \pm}(\tau) d \tau\right)
$$

After the substitution in (1), we obtain $\pm i \hbar \dot{P}_{\vec{k}}^{ \pm}(t)=\left(P_{\vec{k}}^{ \pm}(t)\right)^{2}-\epsilon_{\vec{k}}^{2}(t)$.

We expand $P_{\vec{k}}^{ \pm}$in power series of $\hbar$ thus, $P_{\vec{k}}^{ \pm}(t)=\sum_{n=0}^{\infty} \hbar^{n} P_{n, \vec{k}}^{ \pm}(t)$. We obtain, after having equalized the powers of $\hbar$

$$
P_{0, \vec{k}}^{ \pm}(t)=\epsilon_{\vec{k}}(t) ; \quad P_{1, \vec{k}}^{ \pm}(t)= \pm \frac{i \dot{\epsilon}_{\vec{k}}(t)}{2 \epsilon_{\vec{k}}(t)} ; \quad \text { etc... }
$$

Now, let $\varphi_{W K B, \vec{k}}^{n, \pm}(t)$ be the semi-classical approximation of order $n$ of $\varphi_{\vec{k}}^{ \pm}(t)$, then

$$
\varphi_{W K B, \vec{k}}^{1, \pm}(t)=\frac{1}{\sqrt{2 \epsilon_{\vec{k}}(t)}} \exp \left( \pm \frac{i}{\hbar} \int_{T_{1}}^{t} \epsilon_{\vec{k}}(\tau) d \tau\right) ; \quad \text { etc... }
$$

\subsection{W.K.B. bounds}

Theorem 2.1. If we assume that $\omega_{\vec{k}}(t)$ is monotonous in the interval $\left(T_{1}, T_{2}\right)$. Then, for the differential equation

$$
\ddot{u}_{\vec{k}}+\omega_{\vec{k}}^{2}(t) u_{\vec{k}}=g_{\vec{k}}(t)
$$

we have the following bounds

A) When $\dot{\omega}_{\vec{k}}(t) \geq 0$

$$
\left|u_{\vec{k}}(t)\right| \leq \frac{\hbar}{\epsilon_{\vec{k}}\left(T_{1}\right)} \sqrt{\left|\dot{u}_{\vec{k}}\left(T_{1}\right)\right|^{2}+\frac{\epsilon_{\vec{k}}^{2}\left(T_{1}\right)}{\hbar^{2}}\left|u_{\vec{k}}\left(T_{1}\right)\right|^{2}}+\hbar \int_{T_{1}}^{t} \frac{\left|g_{\vec{k}}(\tau)\right|}{\epsilon_{\vec{k}}(\tau)} d \tau
$$


B) When $\dot{\omega}_{\vec{k}}(t) \leq 0$

$$
\left|u_{\vec{k}}(t)\right| \leq \frac{\hbar}{\epsilon_{\vec{k}}(t)}\left[\sqrt{\left|\dot{u}_{\vec{k}}\left(T_{1}\right)\right|^{2}+\frac{\epsilon_{\vec{k}}^{2}\left(T_{1}\right)}{\hbar^{2}}\left|u_{\vec{k}}\left(T_{1}\right)\right|^{2}}+\hbar \int_{T_{1}}^{t}\left|g_{\vec{k}}(\tau)\right| d \tau\right]
$$

Proof:

A) From the equation (16), we obtain

$$
\frac{1}{\omega_{\vec{k}}^{2}(t)} \frac{d\left|\dot{u}_{\vec{k}}\right|^{2}}{d t}+\frac{d\left|u_{\vec{k}}\right|^{2}}{d t}=\frac{1}{\omega_{\vec{k}}^{2}(t)}\left(g_{\vec{k}}(t) \dot{u}^{*}+g_{\vec{k}}^{*}(t) \dot{u}\right) .
$$

Consequently

$$
\frac{d}{d t}\left(\frac{\left|\dot{u}_{\vec{k}}\right|^{2}}{\omega_{\vec{k}}^{2}(t)}+\left|u_{\vec{k}}\right|^{2}\right)=-\frac{2 \dot{\omega}_{\vec{k}}(t)}{\omega_{\vec{k}}^{3}(t)}\left|\dot{u}_{\vec{k}}\right|^{2}+\frac{1}{\omega_{\vec{k}}^{2}(t)}\left(g_{\vec{k}}(t) \dot{u}^{*}+g_{\vec{k}}^{*}(t) \dot{u}\right) \leq \frac{2\left|g_{\vec{k}}(t)\right|\left|\dot{u}_{\vec{k}}\right|}{\omega_{\vec{k}}^{2}(t)}
$$

From this result we can deduce

$$
\frac{d}{d t} \sqrt{\frac{\left|\dot{u}_{\vec{k}}\right|^{2}}{\omega_{\vec{k}}^{2}(t)}+\left|u_{\vec{k}}\right|^{2}} \leq \frac{\left|g_{\vec{k}}(t)\right|}{\omega_{\vec{k}}(t)} .
$$

Now, after integrating we obtain the result.

B) From the equation (16), we have

$$
\frac{d\left|\dot{u}_{\vec{k}}\right|^{2}}{d t}+\omega_{\vec{k}}^{2}(t) \frac{d\left|u_{\vec{k}}\right|^{2}}{d t}=g_{\vec{k}}(t) \dot{u}^{*}+g_{\vec{k}}^{*}(t) \dot{u}
$$

Consequently,

$$
\frac{d}{d t}\left(\left|\dot{u}_{\vec{k}}\right|^{2}+\omega_{\vec{k}}^{2}(t)\left|u_{\vec{k}}\right|^{2}\right)=2 \dot{\omega}_{\vec{k}}(t) \omega_{\vec{k}}(t)\left|u_{\vec{k}}\right|^{2}+g_{\vec{k}}(t) \dot{u}^{*}+g_{\vec{k}}^{*}(t) \dot{u} \leq 2\left|g_{\vec{k}}(t)\right|\left|u_{\vec{k}}\right|,
$$

and we have

$$
\frac{d}{d t} \sqrt{\left|\dot{u}_{\vec{k}}\right|^{2}+\omega_{\vec{k}}^{2}(t)\left|u_{\vec{k}}\right|^{2}} \leq\left|g_{\vec{k}}(t)\right|
$$

and the result is obtained after integration.

Corollary 2.1. In the case that $\dot{\omega}_{\vec{k}}$ has a finite number of zeros in $\left(T_{1}, T_{2}\right)$. If we assume that $u_{\vec{k}}\left(T_{1}\right)=$ $\dot{u}_{\vec{k}}\left(T_{1}\right)=0$, and we suppose that $\omega_{\vec{k}}$ is a bounded function in $\mathbb{R}$ Then, there exist an adimensional constant $C$ independent of $\hbar, T_{1}, T_{2}$ and $\vec{k}$, such that

$$
\left|u_{\vec{k}}\right| \leq \hbar \frac{C}{\epsilon_{\vec{k}}}\left\|g_{\vec{k}}\right\|_{1} ; \quad\left|\dot{u}_{\vec{k}}\right| \leq C\left\|g_{\vec{k}}\right\|_{1}
$$

where $\epsilon_{\vec{k}}=\sqrt{\frac{4 \pi^{2} c^{2} \hbar^{2}|\vec{k}|^{2}}{L^{2}}+m^{2} c^{4}}$ and $\left\|g_{\vec{k}}\right\|_{1}=\int_{T_{1}}^{T_{2}}\left|g_{\vec{k}}(\tau)\right| d \tau$.

Remark 2.3. The bound is also valid when $\left|T_{2}-T_{1}\right|=+\infty$. 


\section{Application of the W.K.B. method}

Let $\varphi_{\vec{k}}^{ \pm}$be the solutions of (1) in the interval $\left(T_{1}, T_{2}\right)$, that verifies

$$
\left\{\begin{array}{l}
\varphi_{W K B, \vec{k}}^{n, \pm}\left(T_{1}\right)=\varphi_{\vec{k}}^{ \pm}\left(T_{1}\right) \\
\dot{\varphi}_{W K B, \vec{k}}^{n, \pm}\left(T_{1}\right)=\dot{\varphi}_{\vec{k}}^{ \pm}\left(T_{1}\right) .
\end{array}\right.
$$

Due to the corollary 2.1 we have the following bounds

$$
\begin{gathered}
\left|\varphi_{\vec{k}}^{ \pm}(t)-\varphi_{W K B, \vec{k}}^{n, \pm}(t)\right| \leq \frac{\hbar C}{\epsilon_{\vec{k}}}\left\|\left[ \pm \frac{i}{\hbar} \dot{\widetilde{P}}_{n, \vec{k}}^{ \pm}-\frac{1}{\hbar^{2}}\left(\left(\widetilde{P}_{n, \vec{k}}^{ \pm}\right)^{2}-\epsilon_{\vec{k}}^{2}\right)\right] \varphi_{W K B, \vec{k}}^{n, \pm}\right\|_{1} \\
\left|\dot{\varphi}_{\vec{k}}^{ \pm}(t)-\dot{\varphi}_{W K B, \vec{k}}^{n, \pm}(t)\right| \leq C\left\|\left[ \pm \frac{i}{\hbar} \dot{\widetilde{P}}_{n, \vec{k}}^{ \pm}-\frac{1}{\hbar^{2}}\left(\left(\widetilde{P}_{n, \vec{k}}^{ \pm}\right)^{2}-\epsilon_{\vec{k}}^{2}\right)\right] \varphi_{W K B, \vec{k}}^{n, \pm}\right\|_{1}
\end{gathered}
$$

where $\widetilde{P}_{n, \vec{k}}^{ \pm}=\sum_{j=0}^{n} \hbar^{j} P_{j, \vec{k}}^{ \pm}$.

Therefore, if we define

$$
G_{n}\left(\frac{e}{m c^{2}}\right)=\frac{1}{n+1} \sum_{\substack{j_{1}, \ldots, j_{n+1}=0 \\ i_{1}, \ldots, i_{n+1}=1 \\ \sum_{k=1}^{n+1} i_{k} j_{k}=n+1}}^{n+1}\left\|\prod_{k=1}^{n+1}\left(D^{i_{k}} \vec{f}\right)^{j_{k}}\right\|_{1}\left(\frac{e}{m c^{2}}\right)^{\sum_{k=1}^{n+1} j_{k}}
$$

we obtain

$$
\begin{gathered}
\left|\varphi_{\vec{k}}^{ \pm}(t)-\varphi_{W K B, \vec{k}}^{n, \pm}(t)\right| \leq \frac{\hbar^{n} \widetilde{C}}{\left(m c^{2}\right)^{n-2} \epsilon_{\vec{k}}^{\frac{5}{2}}} G_{n}\left(\frac{e}{m c^{2}}\right) \\
\left|\dot{\varphi}_{\vec{k}}^{ \pm}(t)-\dot{\varphi}_{W K B, \vec{k}}^{n, \pm}(t)\right| \leq \frac{\hbar^{n-1} \widetilde{C}}{\left(m c^{2}\right)^{n-2} \epsilon_{\vec{k}}^{\frac{3}{2}}} G_{n}\left(\frac{e}{m c^{2}}\right),
\end{gathered}
$$

where $\widetilde{C}$ is an adimensional constant independent of $\hbar$ and $\vec{k}$.

With these results, we can calculate $\left|b_{\vec{k}}\right|^{2}$. In fact, using the W.K.B. method at the order 2 , we have

$$
\widetilde{P}_{2, \vec{k}}^{ \pm}(t)=\epsilon_{\vec{k}}(t) \pm \frac{i \hbar \dot{\epsilon}_{\vec{k}}(t)}{2 \epsilon_{\vec{k}}(t)}+\frac{\hbar^{2}}{2 \epsilon_{\vec{k}}(t)}\left[-\frac{1}{2} \frac{d}{d t}\left(\frac{\dot{\epsilon}_{\vec{k}}(t)}{2 \epsilon_{\vec{k}}(t)}\right)+\frac{\dot{\epsilon}_{\vec{k}}^{2}(t)}{4 \epsilon_{\vec{k}}^{2}(t)}\right]
$$

We suppose that $\omega_{\vec{k}} \in \mathcal{C}^{2}$ at the point $T_{1}$. In this case

$$
\varphi_{W K B, \vec{k}}^{2, \pm}\left(T_{1}\right)=\phi_{\vec{k}}^{ \pm}\left(T_{1}\right) ; \quad \dot{\varphi}_{W K B, \vec{k}}^{2, \pm}\left(T_{1}\right)=\dot{\phi}_{\vec{k}}^{ \pm}\left(T_{1}\right)
$$

Therefore, if we use the corollary (2.1), the solution of (1) in $\left(T_{1}, T_{2}\right)$ verifies 


$$
\varphi_{\vec{k}}^{ \pm}(t)=\varphi_{W K B, \vec{k}}^{2, \pm}(t)+\hbar^{2} A_{\vec{k}}^{ \pm}(t) ; \quad \dot{\varphi}_{\vec{k}}^{ \pm}(t)=\dot{\varphi}_{W K B, \vec{k}}^{2, \pm}(t)+\hbar B_{\vec{k}}^{ \pm}(t)
$$

with

$$
\left\|A_{\vec{k}}^{ \pm}\right\|_{\infty} \leq \frac{\widetilde{C}}{\epsilon_{\vec{k}}^{\frac{5}{2}}} G_{2}\left(\frac{e}{m c^{2}}\right) ; \quad\left\|B_{\vec{k}}^{ \pm}\right\|_{\infty} \leq \frac{\widetilde{C}}{\epsilon_{\vec{k}}^{\frac{3}{2}}} G_{2}\left(\frac{e}{m c^{2}}\right)
$$

where $\|g\|_{\infty}=\max _{t \in\left(T_{1}, T_{2}\right)}|g(t)|$.

Since $\varphi_{\vec{k}}^{ \pm}$verifies the condition (10), then if we apply the formula (11) we obtain

$$
\left|b_{\vec{k}}\right|^{2}=\frac{\hbar^{2} \dot{\epsilon}_{\vec{k}}^{2}\left(T_{2}\right)}{16 \epsilon_{\vec{k}}^{4}\left(T_{2}\right)}+\hbar^{3} F_{1}+\hbar^{4} F_{2}
$$

with

$$
\begin{gathered}
\left|F_{1}\right| \leq \frac{K}{\epsilon_{\vec{k}}^{4}}\|e \dot{\vec{f}}\|_{\infty}\left[G_{2}\left(\frac{e}{m c^{2}}\right)+\frac{\|e \ddot{\vec{f}}\|_{\infty}}{m c^{2}}+\frac{\|e \dot{\vec{f}}\|_{\infty}^{2}}{\left(m c^{2}\right)^{2}}\right] \\
\left|F_{2}\right| \leq \frac{K}{\epsilon_{\vec{k}}^{4}}\left[G_{2}\left(\frac{e}{m c^{2}}\right)+\frac{\|e \ddot{\vec{f}}\|_{\infty}}{m c^{2}}+\frac{\|e \dot{\vec{f}}\|_{\infty}^{2}}{\left(m c^{2}\right)^{2}}\right]^{2},
\end{gathered}
$$

where $K$ is an adimensional constant.

We see that, in the case that $\omega_{\vec{k}} \notin \mathcal{C}^{1}$ at the point $T_{2}$, then in the semi-classical approximation we obtain

$$
\left|b_{\vec{k}}\right|^{2} \sim \frac{\hbar^{2} \dot{\epsilon}_{\vec{k}}^{2}\left(T_{2}\right)}{16 \epsilon_{\vec{k}}^{4}\left(T_{2}\right)}
$$

Remark 3.1. This result is also valid if we only suppose that $\omega_{\vec{k}} \in \mathcal{C}^{1}$ at the point $T_{1}$. In this case, in order to obtain (38), we have to use the formula (9).

In general, we have the following

Theorem 3.1. If we assume $\omega_{\vec{k}}(t) \in \mathcal{C}^{N+1}$ in $T_{1}, \omega_{\vec{k}}(t) \in \mathcal{C}^{N}$ in $T_{2}$, but $\omega_{\vec{k}}(t) \notin \mathcal{C}^{N+1}$ in $T_{2}$.

Then, in the semi-classical approximation we have

$$
\left|b_{\vec{k}}\right|^{2} \sim \frac{\left.\hbar^{2 N+2} e^{2}\left\|D^{N+1} \vec{f}\right\|\right|_{\infty} ^{2}}{\epsilon_{\vec{k}}^{2 N+4}}
$$




\subsection{Computation of the number of produced pairs}

The theorem (3.1) give a bound of the average number of produced pairs in the $\vec{k}$-sate when $t>T_{2}$. Now, we show a bound of the total average number of produced pairs.

First, we study the following no-physical case. We consider

$$
\omega_{\vec{k}}(t)=\left\{\begin{array}{lll}
\omega_{\vec{k}} & \text { if } & |t|>T \\
\bar{\omega}_{\vec{k}} & \text { if } & |t|<T
\end{array}\right.
$$

In this case

$$
\left|b_{\vec{k}}\right|^{2}=\frac{1}{4} \frac{\left(\epsilon_{\vec{k}}^{2}-\bar{\epsilon}_{\vec{k}}^{2}\right)^{2}}{\epsilon_{\vec{k}}^{2} \bar{\epsilon}_{\vec{k}}^{2}} \sin ^{2}\left(\frac{2}{\hbar} \bar{\epsilon}_{\vec{k}} T\right)
$$

and consequently,

$$
N(t>T) \equiv \sum_{\vec{k} \in \mathbb{Z}^{3}}\left|b_{\vec{k}}\right|^{2}=+\infty
$$

When the hypothesis of the theorem (3.1) are satisfied, then in the semi-classical approximation we have

$$
N\left(t>T_{2}\right) \equiv \sum_{\vec{k} \in \mathbb{Z}^{3}}\left|b_{\vec{k}}\right|^{2} \sim \frac{\hbar^{2 N} \alpha L^{3}\left\|D^{N+1} \vec{f}\right\|_{\infty}^{2}}{\left(m c^{2}\right)^{2 N+1} c^{2}}
$$

where $\alpha=\frac{e^{2}}{\hbar c}$ is the fine structure constant.

In particular, for $N=0$ from (38) we can deduce, in the semi-classical approach, that

$$
N\left(t>T_{2}\right)=\frac{\alpha E\left(T_{2}\right)}{64 m c^{2}}
$$

where $E\left(T_{2}\right)$ is the energy of the field at time $T_{2}$.

In general, using the W.K.B. method, we can prove the following

Theorem 3.2. If we assume that the electric field is $\mathcal{C}^{N}(\mathbb{R} \backslash\{T\})$ and $\mathcal{C}^{N-1}$ in $T$. And we suppose that the field is switched on and off. Then, the average number of produced pairs when the field is switched off, in the semi-classical approximation, is

$$
\frac{\hbar^{2 N} \alpha L^{3}\left\|D^{N+1} \vec{f}\right\|_{\infty}^{2}}{\left(m c^{2}\right)^{2 N+1} c^{2}}
$$

In particular, for $N=0$, this average number is $\frac{\alpha E(T)}{64 m c^{2}}$. 


\section{The Schwinger formula}

In this section we give a rigorous proof of the Schwinger's formula. In order to obtain this formula we consider the potential vector $\vec{f}(t)=(0,0, f(t))$ with

$$
f(t)=\left\{\begin{array}{clc}
-c E T & \text { if } & t<-T \\
c E t & \text { if } & -T<t<T \\
c E T & \text { if } & t>T
\end{array}\right.
$$

where we have supposed that $|T| \gg 1$. In this case $(|T| \gg 1)$ we cannot apply the W.K.B. method, because the function $\omega_{\vec{k}}(t)$ increases to infinity when $T \rightarrow \infty$.

First, we will bound the average number of produced pairs in the $\vec{k}$-state at time $t$. In order to take this bound, we use the diagonalization method in the Schrödinger picture (Haro, 2003). In this picture, the evolution problem is

$$
\left\{\begin{array}{ccc}
i \hbar \partial_{t} \phi & =\hat{H}_{\vec{k}}(t) \phi \\
\phi(-T) & =\phi_{\vec{k}}^{0,0}(-T)
\end{array}\right.
$$

where $\hat{H}_{\vec{k}}(t)=\epsilon_{\vec{k}}(t)\left(\hat{a}_{\vec{k}}^{+}(t) \hat{a}_{\vec{k}}(t)+\hat{b}_{-\vec{k}}^{+}(t) \hat{b}_{-\vec{k}}(t)\right)$ is the diagonalised Hamiltonian, in the Schrödinger picture, at time $t$.

A semi-classical solution of the problem (48) is

$$
\bar{\phi}_{\vec{k}}(t)=\phi_{\vec{k}}^{0,0}(t)-i \hbar \frac{\dot{\epsilon}_{\vec{k}}(t)}{4 \epsilon_{\vec{k}}^{2}(t)} \phi_{\vec{k}}^{1,1}(t)
$$

Now, using (Haro, 2003), it is easy to verifies that

$$
\frac{1}{\hbar} \int_{-T}^{T}\left\|\left(i \hbar \partial_{t}-\hat{H}_{\vec{k}}(t)\right) \bar{\phi}_{\vec{k}}(t)\right\|_{2} d t \leq \min \left\{\frac{2 \pi \hbar c e E}{c^{2} p_{\perp}^{2}+m^{2} c^{4}}, 2 \hbar(c e E)^{2} \int_{-T}^{T} \frac{1}{\epsilon_{\vec{k}}^{3}(\tau)} d \tau\right\}
$$

where $p_{\perp}^{2}=\frac{4 \pi^{2} \hbar^{2}}{L^{2}}\left(k_{1}^{2}+k_{2}^{2}\right)$. We also have $\left|\hbar \frac{\dot{\epsilon}_{\vec{k}}(t)}{4 \epsilon_{\vec{k}}^{2}(t)}\right| \leq \frac{\hbar c e E}{4 \epsilon_{\vec{k}}^{2}(t)}$.

Then, the solution of (48), namely, $\mathcal{T}(t,-T) \phi_{\vec{k}}^{0,0}(-T)$, verifies (Maslov and Fedoriuk, 1981)

$$
\begin{aligned}
& \left\|\mathcal{T}(t,-T) \phi_{\vec{k}}^{0,0}(-T)-\bar{\phi}_{\vec{k}}(t)\right\|_{2} \leq\left\|\phi_{\vec{k}}^{0,0}(-T)-\bar{\phi}_{\vec{k}}(-T)\right\|_{2}+\frac{1}{\hbar} \int_{-T}^{T}\left\|\left(i \hbar \partial_{\tau}-\hat{H}_{\vec{k}}(\tau)\right) \bar{\phi}_{\vec{k}}(\tau)\right\|_{2} d \tau \\
& \leq \min \left\{\frac{3 \pi \hbar c e E}{c^{2} p_{\perp}^{2}+m^{2} c^{4}}, \frac{\hbar c e E}{4 \epsilon_{\vec{k}}^{2}(-T)}+2 \hbar(c e E)^{2} \int_{-T}^{T} \frac{1}{\epsilon_{\vec{k}}^{3}(\tau)} d \tau\right\} \sim O(\hbar) .
\end{aligned}
$$

Now, let $P_{n, \vec{k}}(t)$ be the probability that $n$ pairs are produced in the $\vec{k}$-state at time $t$, then we can deduce

$$
P_{0, \vec{k}}(t)=\mid\left\langle\phi_{\vec{k}}^{0,0}(t), \mathcal{T}(t,-T) \phi_{\vec{k}}^{0,0}(-T)>\left.\right|^{2}=1+O(\hbar)>\frac{1}{2}\right.
$$




$$
\begin{aligned}
P_{1, \vec{k}}(t)= & \left|<\phi_{\vec{k}}^{1,1}(t), \mathcal{T}(t,-T) \phi_{\vec{k}}^{0,0}(-T)>\right|^{2} \leq \min \left\{\frac{10 \pi^{2}(\hbar c e E)^{2}}{\left(c^{2} p_{\perp}^{2}+m^{2} c^{4}\right)^{2}},\right. \\
& \left.\frac{(\hbar c e E)^{2}}{16 \epsilon_{\vec{k}}^{4}(t)}+\frac{(\hbar c e E)^{2}}{16 \epsilon_{\vec{k}}^{4}(-T)}+4 \hbar^{2}(c e E)^{4}\left(\int_{-T}^{T} \frac{1}{\epsilon_{\vec{k}}^{3}(\tau)} d \tau\right)^{2}\right\}
\end{aligned}
$$

Using that (Grib, Mamayev and Mostepanenko, 1994; Marinov and Popov, 1977; Nikishov, 1970)

$$
P_{n, \vec{k}}(t)=P_{0, \vec{k}}(t)\left(1-P_{0, \vec{k}}(t)\right)^{n}=P_{0, \vec{k}}\left(\frac{P_{1, \vec{k}}}{P_{0, \vec{k}}}\right)^{n},
$$

we deduce that the average number of produced pairs in the $\vec{k}$-state at time $t$ is

$$
N_{\vec{k}}(t)=\sum_{n \in \mathbb{Z}} n P_{n, \vec{k}}(t)=\frac{P_{1, \vec{k}}(t)}{P_{0, \vec{k}}^{2}(t)} .
$$

We bound the average number of produced pairs that have the third component of the momentum between $e E t_{1}$ and $e E t_{2}$. Then, using the previous bounds, we obtain

$$
\sum_{\substack{\vec{k} \in \mathbb{Z}^{3} \\ \frac{2 \pi \hbar k_{3}}{L} \in\left[e E t_{1}, e E t_{2}\right]}} N_{\vec{k}}(t) \leq 40 \pi^{3} \frac{L^{3}}{(2 \pi \hbar)^{3}} \frac{(\hbar c e E)^{2}}{\left(m c^{2}\right)^{2} c^{3}} c e E\left(t_{2}-t_{1}\right) .
$$

We also calculate the average number of produced pairs that have the third component of the momentum in $\left(-\infty,-e E\left(T+\sqrt{\frac{m c T}{e E}}\right)\right) \cup\left(e E\left(T+\sqrt{\frac{m c T}{e E}}\right),+\infty\right)$. Then

$$
\begin{aligned}
\sum_{\substack{\vec{k} \in \mathbb{Z}^{3} \\
\frac{2 \pi \hbar\left|k_{3}\right|}{L} \geq e E\left(T+\sqrt{\frac{m c T}{e E}}\right)}} N_{\vec{k}}(t) & \leq \sum_{\vec{k} \in \mathbb{Z}^{3}} \frac{(\hbar c e E)^{2}}{16 \epsilon_{\vec{k}}^{4}(t)}+\sum_{\vec{k} \in \mathbb{Z}^{3}} \frac{(\hbar c e E)^{2}}{16 \epsilon_{\vec{k}}^{4}(-T)} \\
& +4 \hbar^{2}(c e E)^{4} \sum_{\substack{\vec{k} \in \mathbb{Z}^{3} \\
\frac{2 \pi \hbar\left|k_{3}\right|}{L} \geq e E\left(T+\sqrt{\frac{m c T}{e E}}\right.}}\left(\int_{-T}^{T} \frac{1}{\epsilon_{\vec{k}}^{3}(\tau)} d \tau\right)^{2} .
\end{aligned}
$$

The first and second terms are bounded by $\frac{\pi^{2}(\hbar c e E)^{2} L^{3}}{16(2 \pi \hbar)^{3} c^{3} m c^{2}}$.

In order to bound the third term, first we bound

$$
\int_{-T}^{T} \frac{1}{\epsilon_{\vec{k}}^{3}(\tau)} d \tau \leq \frac{1}{\sqrt{c^{2} p_{\perp}^{2}+m^{2} c^{4}}} \int_{-T}^{T} \frac{1}{c^{2} p_{\perp}^{2}+(c e E \tau)^{2}+m^{2} c^{4}} d \tau \leq \frac{\pi}{e E c\left(c^{2} p_{\perp}^{2}+m^{2} c^{4}\right)} .
$$

The third term is less than or equal to 


$$
\begin{aligned}
& (*) \equiv 4 \pi \hbar^{2}(c e E)^{3} \sum_{\substack{\vec{k} \in \mathbb{Z}^{3} \\
\frac{2 \pi \hbar\left|k_{3}\right|}{L} \geq e E\left(T+\sqrt{\frac{m c T}{e E}}\right)}} \frac{1}{c^{2} p_{\perp}^{2}+m^{2} c^{4}} \int_{-T}^{T} \frac{1}{\epsilon_{\vec{k}}^{3}(\tau)} d \tau \\
& =\frac{4 \pi \hbar^{2}(c e E)^{3} L^{3}}{(2 \pi \hbar)^{3}} \int_{-T}^{T} \int_{\mathbb{R}^{2}} \frac{d p_{\perp}}{c^{2} p_{\perp}^{2}+m^{2} c^{4}} \int_{\left|p_{3}\right| \geq e E\left(T+\sqrt{\frac{m c T}{e E}}\right)} \frac{d p_{3}}{\left(c^{2} p_{\perp}^{2}+c^{2}\left(p_{3}+e E \tau\right)^{2}+m^{2} c^{4}\right)^{\frac{3}{2}}} .
\end{aligned}
$$

Now, using the following bound

$$
\begin{aligned}
& \int_{\left|p_{3}\right| \geq e E\left(T+\sqrt{\frac{m c T}{e E}}\right)} \frac{d p_{3}}{\left(c^{2} p_{\perp}^{2}+c^{2}\left(p_{3}+e E \tau\right)^{2}+m^{2} c^{4}\right)^{\frac{3}{2}}} \\
& \leq \frac{1}{m c^{2}} \int_{\left|p_{3}\right| \geq e E\left(T+\sqrt{\frac{m c T}{e E}}\right)} \frac{c\left|p_{3}+e E \tau\right| d p_{3}}{\left(c^{2} p_{\perp}^{2}+c^{2}\left(p_{3}+e E \tau\right)^{2}+m^{2} c^{4}\right)^{\frac{3}{2}}} \\
& \leq \frac{2}{m c^{2}} \frac{1}{\left(c^{2} p_{\perp}^{2}+e E m c^{3} T+m^{2} c^{4}\right)^{\frac{1}{2}}}
\end{aligned}
$$

we obtain

$$
(*) \leq \frac{32 \pi^{2} \hbar^{2}(e E c)^{3} L^{3}}{c^{3} m c^{2}(2 \pi \hbar)^{3}} \frac{T}{\left(m c^{3} e E T\right)^{\frac{1}{4}}\left(m c^{2}\right)^{\frac{1}{2}}} .
$$

Consequently

$$
\sum_{\substack{\vec{k} \in \mathbb{Z}^{3} \\ \frac{2 \pi \hbar\left|k_{3}\right|}{L} \geq e E\left(T+\sqrt{\frac{m c T}{e E}}\right)}} N_{\vec{k}}(t) \leq \frac{\pi^{2}(\hbar c e E)^{2} L^{3}}{8(2 \pi \hbar)^{3} c^{3} m c^{2}}+\frac{32 \pi^{2} \hbar^{2}(e E c)^{3} L^{3}}{c^{3} m c^{2}(2 \pi \hbar)^{3}} \frac{T}{\left(m c^{3} e E T\right)^{\frac{1}{4}}\left(m c^{2}\right)^{\frac{1}{2}}} .
$$

Remark 4.1. These results will be very important in order to compute the average number of produced pairs.

Now we study the problem (Bagrov, Gitman and Shvartsman, 1975; Haro, 2003)

$$
\ddot{u}_{\vec{k}}+\frac{1}{\hbar^{2}}\left(c^{2} p_{\perp}^{2}+c^{2}\left(\frac{2 \pi \hbar k_{3}}{L}+e E t\right)^{2}+m^{2} c^{4}\right) u_{\vec{k}}=0 ; \quad t \in(-T, T) .
$$

If we make the change $y=\sqrt{\frac{2 c}{\hbar e E}}\left(p_{3}+e E t\right)$, then we obtain the differential equation

$$
u_{\vec{k}}^{\prime \prime}+\left(\frac{1}{4} y^{2}-A\right) u_{\vec{k}}=0
$$

where $A=\frac{-1}{2 e E c \hbar}\left(c^{2} p_{\perp}^{2}+m^{2} c^{4}\right)$. 
A independent set of solutions of (52) is

$$
\begin{gathered}
u_{1, \vec{k}}(y)=\exp \left(-\frac{i}{4} y^{2}\right) M\left(-\frac{i}{2} A+\frac{1}{4}, \frac{1}{2}, \frac{i}{2} y^{2}\right) \\
u_{2, \vec{k}}(y)=\frac{1}{\sqrt{2}} \exp \left(-\frac{i}{4} y^{2}\right) y \exp \left(-\frac{i \pi}{4}\right) M\left(-\frac{i}{2} A+\frac{3}{4}, \frac{3}{2}, \frac{i}{2} y^{2}\right)
\end{gathered}
$$

where $M$ is the Kummer's function.

We now define

$$
\begin{gathered}
\varphi_{\vec{k}}^{+}(y)=\frac{\Gamma\left(\frac{3}{2}\right)}{\Gamma\left(\frac{3}{4}+\frac{i}{2} A\right)} u_{1}(y)+\frac{\Gamma\left(\frac{1}{2}\right)}{\Gamma\left(\frac{1}{4}+\frac{i}{2} A\right)} u_{2}(y) \\
\varphi_{\vec{k}}^{-}(y)=-i \frac{\Gamma\left(\frac{3}{2}\right)}{\Gamma\left(\frac{3}{4}-\frac{i}{2} A\right)} u_{1}(y)+\frac{\Gamma\left(\frac{1}{2}\right)}{\Gamma\left(\frac{1}{4}-\frac{i}{2} A\right)} u_{2}(y)
\end{gathered}
$$

Then for $y<0$, we have (Abramowitz and Stegun, 1968)

$$
\begin{gathered}
\varphi_{\vec{k}}^{+}(y)=\bar{A} B \exp \left(\frac{\pi A}{4}\right) \exp \left(-\frac{i \pi}{8}\right)\left(\frac{y^{2}}{2}\right)^{-\frac{1}{4}-\frac{i}{2} A} \exp \left(\frac{i}{4} y^{2}\right)\left[1+R\left(A, y^{2}\right)\right] \\
\varphi_{\vec{k}}^{-}(y)=-\bar{A} B \exp \left(\frac{\pi A}{4}\right) \exp \left(\frac{i \pi}{8}\right)\left(\frac{y^{2}}{2}\right)^{-\frac{1}{4}+\frac{i}{2} A} \exp \left(-\frac{i}{4} y^{2}\right)\left[1+R\left(A, y^{2}\right)\right]
\end{gathered}
$$

with

$$
\bar{A}=\frac{\Gamma\left(\frac{1}{2}\right) \Gamma\left(\frac{3}{2}\right)}{\Gamma\left(\frac{1}{4}+\frac{i}{2} A\right) \Gamma\left(\frac{3}{4}+\frac{i}{2} A\right)} ; \quad B=\frac{\Gamma\left(\frac{1}{4}+\frac{i}{2} A\right)}{\Gamma\left(\frac{1}{4}-\frac{i}{2} A\right)}+i \frac{\Gamma\left(\frac{3}{4}+\frac{i}{2} A\right)}{\Gamma\left(\frac{3}{4}-\frac{i}{2} A\right)} .
$$

In order to obtain the bound of the function $R\left(A, y^{2}\right)$ we have used (Nikiforov and Ouvarov, 1976), and we have obtained

$$
\left|R\left(A, y^{2}\right)\right| \leq-K \frac{A}{y^{2}} \exp \left(-\frac{A \pi}{2}\right),
$$

where $K$ is a positive adimensional constant independent of $A$ and $y$.

For $y>0$, we have

$$
\begin{aligned}
\varphi_{\vec{k}}^{+}(y)= & \exp \left(\frac{\pi A}{4}\right)\left\{2 \bar{A} \exp \left(\frac{i \pi}{8}\right)\left(\frac{y^{2}}{2}\right)^{-\frac{1}{4}+\frac{i}{2} A} \exp \left(\frac{-i y^{2}}{4}\right)\left[1+R\left(A, y^{2}\right)\right]\right. \\
& \left.+\bar{A} C \exp \left(\frac{-i \pi}{8}\right)\left(\frac{y^{2}}{2}\right)^{-\frac{1}{4}-\frac{i}{2} A} \exp \left(\frac{i y^{2}}{4}\right)\left[1+R\left(A, y^{2}\right)\right]\right\}
\end{aligned}
$$




$$
\begin{aligned}
\varphi_{\vec{k}}^{-}(y)= & \exp \left(\frac{\pi A}{4}\right)\left\{2 i \bar{A}^{*} \exp \left(\frac{-i \pi}{8}\right)\left(\frac{y^{2}}{2}\right)^{-\frac{1}{4}-\frac{i}{2} A} \exp \left(\frac{i y^{2}}{4}\right)\left[1+R\left(A, y^{2}\right)\right]\right. \\
& \left.+\bar{A} C \exp \left(\frac{i \pi}{8}\right)\left(\frac{y^{2}}{2}\right)^{-\frac{1}{4}+\frac{i}{2} A} \exp \left(\frac{-i y^{2}}{4}\right)\left[1+R\left(A, y^{2}\right)\right]\right\}
\end{aligned}
$$

with

$$
C=\frac{\Gamma\left(\frac{1}{4}+\frac{i}{2} A\right)}{\Gamma\left(\frac{1}{4}-\frac{i}{2} A\right)}-i \frac{\Gamma\left(\frac{3}{4}+\frac{i}{2} A\right)}{\Gamma\left(\frac{3}{4}-\frac{i}{2} A\right)} .
$$

Remark 4.2. For the derivate we obtain expressions similar to (57),..,(60).

Now, we study the case $\frac{2 \pi \hbar\left|k_{3}\right|}{e E L} \leq T-\sqrt{\frac{T m c}{e E}}$. Since $y=\sqrt{\frac{2 c}{\hbar e E}}\left(p_{3}+e E t\right)$ we have $y(-T)<0$ and $y(T)>0$. Therefore, from the formula (9) and the expressions (57), (58), (59) and (60) it is easy to see that for $\frac{2 \pi \hbar\left|k_{3}\right|}{e E L} \leq T-\sqrt{\frac{T m c}{e E}}$, we have

$$
\lim _{T \rightarrow \infty}\left|b_{\vec{k}}\right|^{2}=\frac{|C|^{2}}{|B|^{2}}(1+G(\vec{p}, T))=\exp \left(-\frac{\pi}{e E c \hbar}\left(c^{2} p_{\perp}^{2}+m^{2} c^{4}\right)\right)+F(\vec{p}, T),
$$

with

$$
|F(\vec{p}, T)| \leq \widetilde{K} \frac{c^{2} p_{\perp}^{2}+m^{2} c^{4}}{m c^{3} T e E} \exp \left(-\frac{3 \pi}{4 e E c \hbar}\left(c^{2} p_{\perp}^{2}+m^{2} c^{4}\right)\right)
$$

where $\widetilde{K}$ is an adimensional constant independent of $T, p_{\perp}$ and $\hbar$.

With these results we can calculate the average number of produced pairs per unit time and unit volume, when $T \rightarrow \infty$.

$$
\begin{aligned}
& \sum_{\vec{k} \in \mathbb{Z}^{3}} \frac{\left|b_{\vec{k}}\right|^{2}}{2 T L^{3}}=\sum_{\vec{k} \in \mathbb{Z}^{3}} \frac{\left|b_{\vec{k}}\right|^{2}}{2 T L^{3}}+\sum_{\vec{k} \in \mathbb{Z}^{3}} \frac{\left|b_{\vec{k}}\right|^{2}}{2 T L^{3}} \\
& \frac{2 \pi \hbar\left|k_{3}\right|}{L} \geq e E\left(T+\sqrt{\frac{T m c}{e E}}\right) \quad \quad \frac{2 \pi \hbar\left|k_{3}\right|}{L} \leq e E\left(T-\sqrt{\frac{T m c}{e E}}\right) \\
& +\sum_{\substack{\vec{k} \in \mathbb{Z}^{3} \\
e E\left(T-\sqrt{\frac{T m c}{c c}} \leq \frac{2 \pi \hbar\left|k_{3}\right|}{L} \leq e E\left(T+\sqrt{\frac{T m c}{c c}}\right)\right.}} \frac{\left|b_{\vec{k}}\right|^{2}}{2 T L^{3}} .
\end{aligned}
$$

Then, due to the expressions (49) and (50), we have

$$
\lim _{T \rightarrow \infty} \sum_{\substack{\vec{k} \in \mathbb{Z}^{3} \\ e E\left(T-\sqrt{\frac{T m c}{e E}}\right) \leq \frac{2 \pi \hbar\left|k_{3}\right|}{L} \leq e E\left(T+\sqrt{\frac{T m c}{e E}}\right)}} \frac{\left|b_{\vec{k}}\right|^{2}}{2 T L^{3}} \leq \lim _{T \rightarrow \infty} 40 \pi^{3} \frac{1}{(2 \pi \hbar)^{3}} \frac{(\hbar c e E)^{2}}{\left(m c^{2}\right)^{2} c^{3}} \sqrt{\frac{e E m c^{3}}{T}}=0 .
$$




$$
\lim _{T \rightarrow \infty} \sum_{\substack{k_{3} \in \mathbb{Z} \\ \frac{2 \pi \hbar\left|k_{3}\right|}{L} \geq e E(T+\sqrt{T})}} \frac{\left|b_{\vec{k}}\right|^{2}}{2 T L^{3}} \leq \lim _{T \rightarrow \infty}\left[\frac{\pi^{2}(\hbar c e E)^{2}}{16 T(2 \pi \hbar)^{3} c^{3} m c^{2}}+\frac{16 \pi^{2} \hbar^{2}(e E c)^{3}}{c^{3} m c^{2}(2 \pi \hbar)^{3}} \frac{1}{\left(m c^{3} e E T\right)^{\frac{1}{4}}\left(m c^{2}\right)^{\frac{1}{2}}}\right]=0 .
$$

And, due to the formula (61), we obtain

$$
\begin{aligned}
\lim _{T \rightarrow \infty} \sum_{\substack{\vec{k} \in \mathbb{Z}^{3} \\
\frac{2 \pi \hbar\left|k_{3}\right|}{L} \leq e E\left(T-\sqrt{\frac{T m c}{e E}}\right)}} \frac{\left|b_{\vec{k}}\right|^{2}}{2 T L^{3}} & =\lim _{T \rightarrow \infty} \frac{2\left(T-\sqrt{\frac{T m c}{e E}}\right)}{2 T(2 \pi \hbar)^{3}} \int_{\mathbb{R}^{2}} \exp \left(-\frac{\pi\left(c^{2} p_{\perp}^{2}+m^{2} c^{4}\right)}{\hbar c e E}\right) d p_{\perp} \\
& =\frac{E^{2} \alpha}{8 \pi^{3} \hbar} \exp \left(-\frac{\pi m^{2} c^{4}}{\hbar c e E}\right) .
\end{aligned}
$$

Consequently, we have proved that

$$
\lim _{T \rightarrow \infty} \sum_{\vec{k} \in \mathbb{Z}^{3}} \frac{\left|b_{\vec{k}}\right|^{2}}{2 T L^{3}}=\frac{E^{2} \alpha}{8 \pi^{3} \hbar} \exp \left(-\frac{\pi m^{2} c^{4}}{\hbar c e E}\right)
$$

Remark 4.3. In (Holstein, 1999; Nikishov, 1970) the authors calculate the quantity

$$
\frac{1}{2 T L^{3}} \sum_{\vec{k} \in \mathbb{Z}^{3}} \lim _{T \rightarrow \infty}\left|b_{\vec{k}}\right|^{2}=\frac{1}{2 T(2 \pi \hbar)^{3}} \int_{\mathbb{R}^{3}} \exp \left(-\frac{\pi\left(c^{2} p_{\perp}^{2}+m^{2} c^{4}\right)}{\hbar c e E}\right) d \vec{p}
$$

and make the replacement $\int_{\mathbb{R}} d p_{3} \rightarrow 2 e E T$ in order to obtain the formula (63). Clearly, this argument is meaningless.

Using the same argument, we can prove that, when $T \rightarrow \infty$, the relative probability that a pair is produced per unit time and unit volume, is (Haro, to appear)

$$
\lim _{T \rightarrow \infty} \sum_{\vec{k} \in \mathbb{Z}^{3}} \frac{1}{2 T L^{3}} \frac{\left|b_{\vec{k}}\right|^{2}}{\left|a_{\vec{k}}\right|^{2}}=\frac{E^{2} \alpha}{8 \pi^{3} \hbar} \sum_{n=1}^{\infty} \frac{(-1)^{n+1}}{n} \exp \left(-\frac{n \pi m^{2} c^{4}}{\hbar c e E}\right),
$$

in contrast with the interpretation given by Schwinger and others authors (Greiner, Müller and Rafelski, 1985; Itzykson and Zuber, 1980; Popov, 1972; Schwinger, 1951).

Acknowledgments: This paper is partially supported by the project BFM2002-04613-C03-01 of the MCyT, Spain. 


\section{References}

1 M.ABRAMOWITZ, J.STEGUN, Handbook of Mathematical Functions; National Bureau of Standards, Washington DC (1968).

2 V.G.BAGROV, D.M.GITMAN, SH.M. SHVARTSMAN, Concerning the production of electron-positron pairs from vacuum; Zh.Eksp.Teor.Fiz.68, page 392-399 (1975).

3 M.V.BERRY, Semiclassically weak reflection above analytic and no-analytic potential barriers; J.Phys.A: Math.Gen.15, page 3693-3704 (1982).

4 J.M.EISENBERG, G.KÄLBERMANN, Pair production in transport equations; Physical Review D35 no.5, page 368-375 (1988).

5 S.A.FULLING, Aspects of Quantum Field Theory in Curved Space-Time; London Mathematical Society Student Text 17 (1985).

6 W.GREINER, B.MÜLLER, J.RAFELSKI, Quantum Electrodynamics of Strong Fields; Springer-Verlag (1985).

7 A.A.GRIB, S.G.MAMAYEV, V.M.MOSTEPANENKO, Vacuum Quantum Effects in Strong Fields; Publishing Board Laboratory for Theoretical Physics, St. Petersburg (1994).

8 J.HARO, Pair production in a uniform electric field; Int. Jour. Theor. Phys.42, no.3, page 531-547, (2003).

9 J.HARO, The Schwinger formula revisited; Int. Jour. Theor. Phys., (to appear).

10 B.R. HOLSTEIN, Strong field pair production; Am. J. Phys. 67, no.6, page 499-507, (1999).

11 C.ITZYKSON, J.B.ZUBER, Quantum field theory; McGraw-Hill International Editions, (1980).

12 S.M.MARINOV, V.S.POPOV, Electron-Positron Pair Creation from Vacuum Induced by Variable Electric Field; Fortschritte der Physik 25, page 373-400 (1977).

13 V.P. MASLOV, M.V.FEDORIUK, Semi-Classical Approximation in Quantum Mechanics; D. Riedel Publishing Company, Dordrecht, Holland (1981).

14 A.NIKIFOROV, V.OUVAROV, Éléments de la Théorie des fonctions spéciales; Editons Mir (1976).

15 A.I..NIKISHOV, Pair production by a constant electric field; Soviet Physics JETP 30, no.4, page 660-662 (1970).

16 A.I..NIKISHOV, Barrier scattering in field theory removal of Klein paradox; Nuclear Physics B21, page 346-358 (1970).

17 V.S.POPOV, Pair production in a variable external field (Quasi-classical approximation); Sov. Phys. JETP 34, page 709-718 (1972).

18 J.S.SCHWINGER, On Gauge Invariance and Vacuum Polarisation; Physical Review 82, no.5, page 664679, (1951). 